\title{
Cocaine use worsens coronary atherosclerosis in HIV infected
}

\author{
Richard A. P. Takx ${ }^{1}$ (D) Csilla Celeng $^{1}$ \\ Received: 18 December 2020 / Revised: 20 January 2021 / Accepted: 17 February 2021 / Published online: 8 March 2021 \\ (C) European Society of Radiology 2021
}

Kolossváry et al [1] investigated the difference in annual atherosclerosis progression rate between $226 \mathrm{HIV}$-infected and 74-uninfected African Americans using volumetric quantification on contrast-enhanced CT. The mean plaque volume increased from $34.8 \mathrm{~mm}^{3}$ (Q1-Q3 $13.2-101.7 \mathrm{~mm}^{3}$ ) at baseline to $74.9 \mathrm{~mm}^{3}\left(\mathrm{Q} 1-\mathrm{Q} 330.8-176.6 \mathrm{~mm}^{3}\right)$ at follow-up (mean follow-up $4.0 \pm 2.3$ years). No significant difference was found between HIV-infected and HIV-uninfected individuals. Each percentage of atherosclerotic cardiovascular disease (ASCVD) risk and each year of cocaine use were associated with a significant increase in plaque volumes in HIVinfected individuals, but not in HIV-uninfected people. Their study suggests that HIV infection indirectly promotes plaque volume progression.

HIV/AIDS has become a chronic inflammatory disease with the aid of antiretroviral therapy (ART). ART in itself can worsen blood lipid levels. Hence, potential cardiovascular complications of HIV infection and therapy should be taken into account and managed [2]. By the year 2030, it is anticipated that $73 \%$ of people with HIV infection will be 50 years or older and $78 \%$ will have cardiovascular diseases [3]. Inflammation precipitated by elevated lipid levels plays an important pathobiological role in the development of atherosclerosis [4]. Patients with HIV managed with ART have more atherosclerosis and a greater non-calcified plaque burden due to a chronic inflammatory state [5]. This is reflected by the observation that patients with HIV are on average 10 years younger when they develop acute coronary syndrome [6]. Nevertheless, the precise mechanism remains to be elucidated. In an FDG PET CT study, it was observed that both lymph node and arterial activity were increased in those infected with HIV, but only lymph node activity correlated with markers of viral disease (viral load, CD8+ T cells, and CD4/

Richard A. P. Takx

r.a.p.takx@umcutrecht.nl

1 Department of Radiology, UMC Utrecht, Heidelberglaan 100, P.O. Box 85500, 3584, CX Utrecht, The Netherlands
CD8 ratio) [7] suggesting that lymph node and arterial inflammation have different pathways of immune activation.

Contrary to the chronic inflammatory theory, Kolossváry et al [1] showed no link between HIV infection and coronary plaque burden in this longitudinal study of African Americans. Nevertheless, they found that each year of cocaine use in HIVinfected individuals was associated with a greater plaque burden. They also found that HIV modifies the effect of cardiovascular risk factors in a negative manner, meaning each percentage increase in ASCVD risk is associated with plaque volume progression. Another recent publication by Lai et al [8] in African Americans with and without HIV infection showed also that cocaine use was independently associated with coronary atherosclerosis, especially in individuals on ART. Lai et al found similarly no association between HIV infection and subclinical CAD. Management should consist of cardiovascular risk factor modification including abstaining from cocaine. Also, medical therapy can be considered. Angiotensinconverting enzyme (ACE) inhibitors are cardiovascular protective by lowering blood pressure and improving endothelial cell function. Moreover, ACE inhibitors have the potential to reduce cocaine use by altering levels of dopamine and corticotropin-releasing factor in the brain [9]. Dopaminergic drugs may reduce the use of cocaine by compensating for downregulation of dopamine due to long-term cocaine use. Corticotropin-releasing factor may mediate dysphoria associated with abstinence and also stress-related relapse [9].

In conclusion, adequate cardiovascular management (including medical therapy) in combination with abstaining from cocaine use is warranted in HIV-infected individuals, especially African Americans. These observations still have to be confirmed in different ethnic/racial groups. CTA can be a useful tool for detecting and monitoring subclinical atherosclerosis.

Funding The authors state that this work has not received any funding.

\section{Declarations}

Guarantor The scientific guarantor of this publication is Richard A.P. Takx. 
Conflict of interest The authors of this manuscript declare no relationships with any companies whose products or services may be related to the subject matter of the article.

Statistics and biometry No complex statistical methods were necessary for this paper.

Informed consent Written informed consent was not required for this study because it is an editorial.

Ethical approval Institutional Review Board approval was not required because it is an editorial.

\section{Methodology}

- editorial

\section{References}

1. Kolossváry M, Fishman EK, Gerstenblith G et al (2021) Cardiovascular risk factors and illicit drug use may have a more profound effect on coronary atherosclerosis progression in people living with HIV. Eur Radiol. https://doi.org/10.1007/s00330-02107755-7

2. Hsue PY, Waters DD (2019) HIV infection and coronary heart disease: mechanisms and management. Nat Rev Cardiol 16:745-759
3. Smit M, Brinkman K, Geerlings S et al (2015) Future challenges for clinical care of an ageing population infected with HIV: a modelling study. Lancet Infect Dis 15:810-818

4. Wolf D, Ley K (2019) Immunity and inflammation in atherosclerosis. Circ Res 124:315-327

5. D'Ascenzo F, Cerrato E, Calcagno A et al (2015) High prevalence at computed coronary tomography of non-calcified plaques in asymptomatic HIV patients treated with HAART: a meta-analysis. Atherosclerosis 240:197-204

6. Hsue PY, Giri K, Erickson S et al (2004) Clinical features of acute coronary syndromes in patients with human immunodeficiency virus infection. Circulation 109:316-319

7. Tawakol A, Ishai A, Li D et al (2017) Association of arterial and lymph node inflammation with distinct inflammatory pathways in human immunodeficiency virus infection. JAMA Cardiol 2:163-171

8. Lai H, Moore R, Celentano DD et al (2016) HIV infection itself may not be associated with subclinical coronary artery disease among African Americans without cardiovascular symptoms. J Am Heart Assoc 5:e002529

9. Margolin A, Avants SK, Setaro JF, Rinder HM, Grupp L (2000) Cocaine, HIV, and their cardiovascular effects: is there a role for ACE-inhibitor therapy? Drug Alcohol Depend 61:35-45

Publisher's note Springer Nature remains neutral with regard to jurisdictional claims in published maps and institutional affiliations. 